\title{
Aplicación de herramientas de control de calidad en una pequeña panadería. Un estudio de caso
}

\section{Quality control application in a Small Bakery. A Case study}

\author{
DOI: http://doi.org/10.17981/bilo.2.1.2020.6
}

Artículo de Investigación Científica. Fecha de Recepción: 30/05/2020. Fecha de Aceptación: 13/06/2020

\author{
Zaskhia Marin-Pabón \\ Universidad de la Costa CUC, Barranquilla, (Colombia) \\ zmarin@cuc.edu.co \\ Luz Olmos-Estrada \\ Universidad de la Costa CUC, Barranquilla, (Colombia) \\ lolmos5@cuc.edu.co \\ Ana de los Reyes- Royero \\ Universidad de la Costa CUC, Barranquilla, (Colombia) \\ adelosre4@cuc.edu.co \\ Geraldine Lobo-Osorio \\ Universidad de la Costa CUC, Barranquilla, (Colombia) \\ globo@cuc.edu.co \\ Rafael Ruiz-Carmona \\ Universidad de la Costa CUC, Barranquilla, (Colombia) \\ rruiz13@cuc.edu.co \\ Alexander Troncoso-Palacio \\ Universidad de la Costa CUC, Barranquilla, (Colombia) \\ atroncos1@cuc.edu.co
}

Para citar este artículo:

Z. Marin-Pabón, L. Olmos-Estrada, A. De los Reyes Royero, G. Lobo Osorio, R. Ruiz Carmona, A. Troncoso-Palacio, "Aplicación de herramientas de control de calidad en una pequeña panadería. Un estudio de caso", BILO, vol. 2, no. 1, 2020. DOI: http://doi.org/10.17981/bilo.2.1.2020.6

\section{Resumen}

En los negocios pequeños de panadería es fundamental mantener la clientela satisfecha si por cualquier motivo disminuye el número de clientes, se verá afectada económicamente lo que haría que al transcurrir del tiempo tienda a desaparecer del mercado ocasionando pérdidas económicas. Razón por la cual se inició este estudio donde se pueden evidenciar los factores que influyen negativamente en el proceso de elaboración del pan, a través de la utilización de las herramientas de calidad como la carta de control XR, el diagrama Ishikawa y el , diagrama de Pareto, para observar detalladamente las posibles causas de pérdida de clientes en una pequeña panadería ubicada en el municipio de Sabanalarga-Atlántico, 
Colombia, llamada ROYALIZ, donde se encontraron tres principales causas de pérdida de tiempo al momento de elaborar el pan que son tiempo en el horneado, mezcla no adecuada y mal estado de las herramientas de elaboración las cuales han conducido a la disminución de clientes. Por lo cual se propone implementar tácticas para atraer nuevamente a los clientes tales como nuevos productos creando un ambiente atractivo, asimismo, se ejecutaron estrategias como el cambio de la maquinaria (hornos), mantenimientos de los mismos, inspección en el tiempo para que los productos no tengan defectos y así llevar un buen manejo en el proceso de supervisión al momento de la elaboración del pan.

Palabras clave: Elaboración de pan; Herramientas de Calidad; Cartas de Control; Diagrama Ishikawa; Estrategias de Control; Supervisión.

\begin{abstract}
In small bakery businesses it is essential to keep customers satisfied if for any reason the number of customers decreases, it will be economically affected, which would cause it to disappear from the market over time causing economic losses. Reason why this study started where the factors that negatively influence the bread making process can be evidenced, through the use of quality tools such as the XR control chart, the Ishikawa diagram and the Pareto, to observe in detail the possible causes of loss of customers in a small bakery located in the municipality of Sabanalarga-Atlántico, Colombia, called ROYALIZ, where three main causes of loss of time were found when making bread that are time in baking, improper mixing and poor condition of the processing tools which have led to a decrease in customers. Therefore, it is proposed to implement tactics to attract customers again, such as new products, creating an attractive environment. Likewise, strategies such as changing machinery (ovens), maintenance thereof, and inspection over time for products have no defects and thus have good management in the supervision process at the time of making the bread.
\end{abstract}

Keywords: Bread making; Quality tools; Control Charts; Ishikawa Diagram; Control Strategies; Supervision.

\title{
I. INTRODUCCIÓN
}

Las panaderías a nivel mundial son consideradas como uno de los negocios prioritarios para el sustento diario, ya que se encuentran en distintas partes del mundo, en ellas son elaborados alimentos como el pan, por el que muchas personas estas dispuestas a invertir pues hace parte del consumo de muchas familias, sin embargo, en ellas pueden ocurrir imprevistos. No obstante, en todo proceso productivo es necesario inspeccionar cada paso que se realiza, puesto que los problemas de calidad pueden aparecer generando pérdidas; en una panadería normalmente se presentan inconvenientes en el momento de elaboración de pan, ya sea en sus tiempos de horneado, personal sin experiencia, incluso en la atención a los clientes. Hoy en día muchas empresas se preocupan por su futuro y buscan la mejor manera de mantenerse en el mercado. En el estudio realizado a ROYALIZ, una microempresa del municipio de Sabanalarga-Atlántico, se encontraron perdidas a nivel de clientes por no tener estándares de calidad, especialmente por no tener en cuenta que en el desarrollo de cualquier actividad existen variaciones y difícilmente se pueden evitar; en este sentido se propuso establecer un plan de acción basado en las herramientas básicas de control para mejorar los resultados; tales como, gráficos de control para verificar el comportamiento de los procesos y el diagrama Ishikawa para analizar las causas del problema y buscar la mejor solución posible, entre otros.

\section{REVISION LITERARIA}

La seguridad alimentaria es un aspecto prioritario para todos los profesionales y constituye una demanda expresa de la sociedad actual. Por otra parte, las panaderías deben poner en el mercado productos seguros para el consumidor, según establece la legislación vigente. Por todo ello las panaderías, al igual que el resto de industrias, deben adaptar sus condiciones de producción a la implantación de sistemas eficaces de autocontrol que garanticen la seguridad de los alimentos que elaboran, tal y como exige la legislación de la Unión Europea, basados en la aplicación del Sistema de Análisis de Peligros y Puntos de Control Críticos [1]. El pan se elabora desde tiempos remotos por lo que es indispensable en la alimentación humana. Su elaboración siempre ha sido un arte, antes lo hacían pocas familias, el amasado mecanizado hace común su procesamiento. Actualmente el pan, lo hacen en todos los lugares del mundo, pero hasta hoy todavía existen muchos secretos en su elaboración. En las últimas décadas se da fundamento científico a la panificación, con el estudio del trigo y sus componentes, determinando que la acción de la GLIADINA y la GLUTENINA son los responsables del esponjamiento de la masa. El consumo del pan [2], cubre un porcentaje significativo en la dieta alimentaria de los hombres, llegando hasta un 25 a 30\% en el caso de Europa y América del Sur, consumiéndose más en la zona urbana. El análisis de causas raíz es un proceso secuencial de preguntas estructuradas para descubrir errores latentes subyacentes en un suceso centinela, por lo que supone una revisión exhaustiva de los elementos que lo integran personas, equipos, procedimientos, información, entornos, contingencias externas. [3], Se recurrió a la utilización de la herramienta de Pareto, Diagrama de Pareto, donde se reconoce que más de $80 \%$ de la problemática en una organización es por causas comunes, es decir, se debe a problemas o situaciones que actúan de manera permanente sobre los procesos. Pero, además, en todo proceso son pocos los problemas o situaciones vitales que contribuyen en gran medida a la problemática global 
de un proceso o una empresa. Lo anterior es la premisa del diagrama de Pareto, cuyo campo de análisis o aplicación son los datos categóricos, y tiene como objetivo ayudar a localizar el o los problemas vitales, así como sus principales causas. La idea es que cuando se quiere mejorar un proceso o atender sus problemas, no se den "palos de ciego" y se trabaje en todos los problemas al mismo tiempo atacando todas sus causas a la vez, sino que, con base en los datos e información aportados por un análisis estadístico, se establezcan prioridades y se enfoquen los esfuerzos donde éstos tengan mayor impacto [4].

El uso conjunto de gráficos de control de media y varianza ha sido extenso estudiado para monitorear procesos que pueden estar sujetos a diferentes tipos de causas asignables que cambian la media de los procesos y aumentan su variabilidad. [5] En los que se presenta un enfoque económico para el gráfico de control X R. [6] Que analiza el uso de la optimización en la definición de los límites de control del gráfico de control X R , con el objetivo de minimizar el tiempo para detectar un cambio en los parámetros [7]. El enfoque comportamental de la lealtad está basado específicamente en la repetición de compra, es decir, si la marca satisface las expectativas del consumidor aumentará la probabilidad de seguir adquiriéndola y resultará difícil arriesgarse a comprar otra marca. En este marco de ideas, los procesos internos - formación de actitudes y compromiso - no tienen relevancia y se limita a considerar solo las compras repetidas. Así, la lealtad puede tener una orientación conductista, es decir, basada en patrones de comportamiento aprendidos, estímulos relacionados con la experiencia y referencia previa (perspectiva estímulo-respuesta). De esta forma, si se conocen los estímulos que afectan el comportamiento de los consumidores, se podrá predecir su lealtad. Ahora bien, dada la multiplicidad de estímulos que pueden influir en el consumidor, se intuye difícil la idea de poder predecir efectivamente [8]. Cabe mencionar, que la empresa que aplique controles internos en sus operaciones, conducirá a conocer la situación real de las mismas, es por ello la importancia de tener una aplicación que sea capaz de verificar que los controles se cumplan para darle una mejor visión sobre su administración. [9]-[10]

\section{METODOLOGIA}

Para realizar la presente investigación se siguió una metodología analítica para conocer las causas de la problemática de disminución de clientes en una pequeña panadería. Para lo cual, se elaboró un diagrama 6 m, luego se procede a realizar la recopilación, tabulación y grafico de datos a través de la herramienta Diagrama de Pareto con el fin de conocer e identificar los pocos vitales en los que se deberían tomar acciones correctivas. Lo cual se evidencia en el siguiente gráfico.

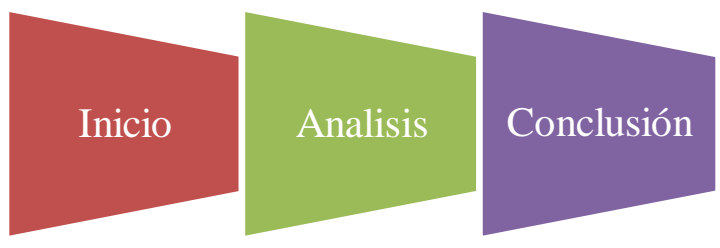

Figura 1. Secuencia metodológica

\section{RESULTADOS}

Se llevó a cabo la recolección de datos necesarios para realizar el diagrama de Ishikawa, en donde se agruparán en seis ramas principales $(6 \mathrm{M})$, Se utiliza este diagrama ya que relaciona el problema con los factores que posiblemente los genera. Como se logra observar se encuentran las distintas causas de la elaboración de los panes.
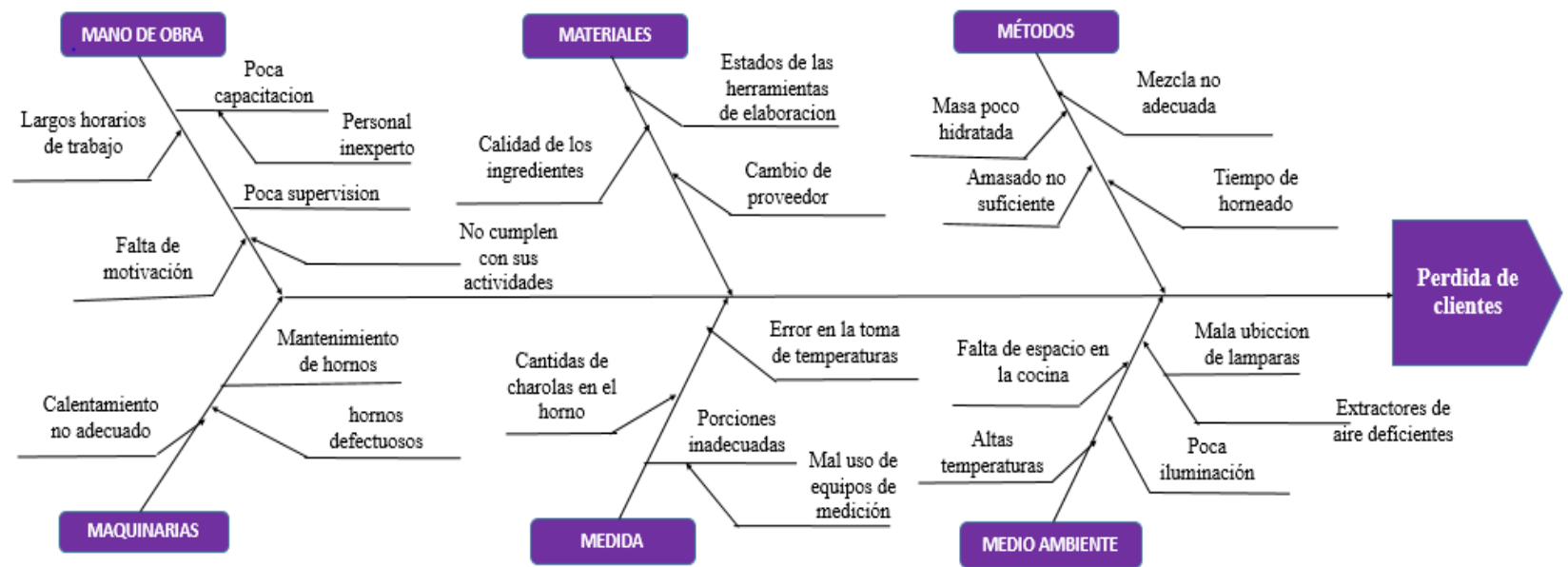
Figura 2. Diagrama Causa-Efecto Pérdida de clientes en panadería ROYALIZ.

Para establecer cuáles son los factores que generan el problema de la pérdida de clientes, se procedió a realizar el diagrama de Pareto, para identificar los vitales y los triviales que afectan la producción de panes en la panadería Royaliz, a cada factor se le asignó un identificador para reconocerlos fácilmente en la gráfica.

\begin{tabular}{cc}
\hline Causa / Problema / Fenómeno & $\begin{array}{c}\text { Datos } \\
\text { recolectados }\end{array}$ \\
\hline Tiempo de horneado & 50 \\
Estados de las herramientas de elaboración & 45 \\
Calentamiento no adecuado & 41 \\
Largos horarios de trabajo & 3 \\
Falta de motivación & 3 \\
Cambio de proveedor & 2 \\
Calidad de los ingredientes & 2 \\
No cumplen con sus actividades & 2 \\
Poca supervisión & 2 \\
Personal inexperto & 2 \\
Poca capacitación & 2 \\
Mantenimientos de hornos & 2 \\
Mal uso de equipos de medición & 2 \\
Mezcla no adecuada & 1 \\
Masa poco hidratada & 1 \\
Amasado no suficiente & 1 \\
Hornos defectuosos & 1 \\
Cantidad de charolas en el horno & 1 \\
Toma de temperaturas en el momento indebido & 1 \\
Porciones inadecuadas & 1 \\
Altas temperaturas & 1 \\
Falta de espacio en la cocina & 1 \\
Mala ubicación de lámparas & 1 \\
Poca iluminación & 1 \\
Extractores de aire insuficientes & 1 \\
\hline Tabla 1. Daos defico Parto
\end{tabular}

Tabla 1. Datos del gráfico de Pareto

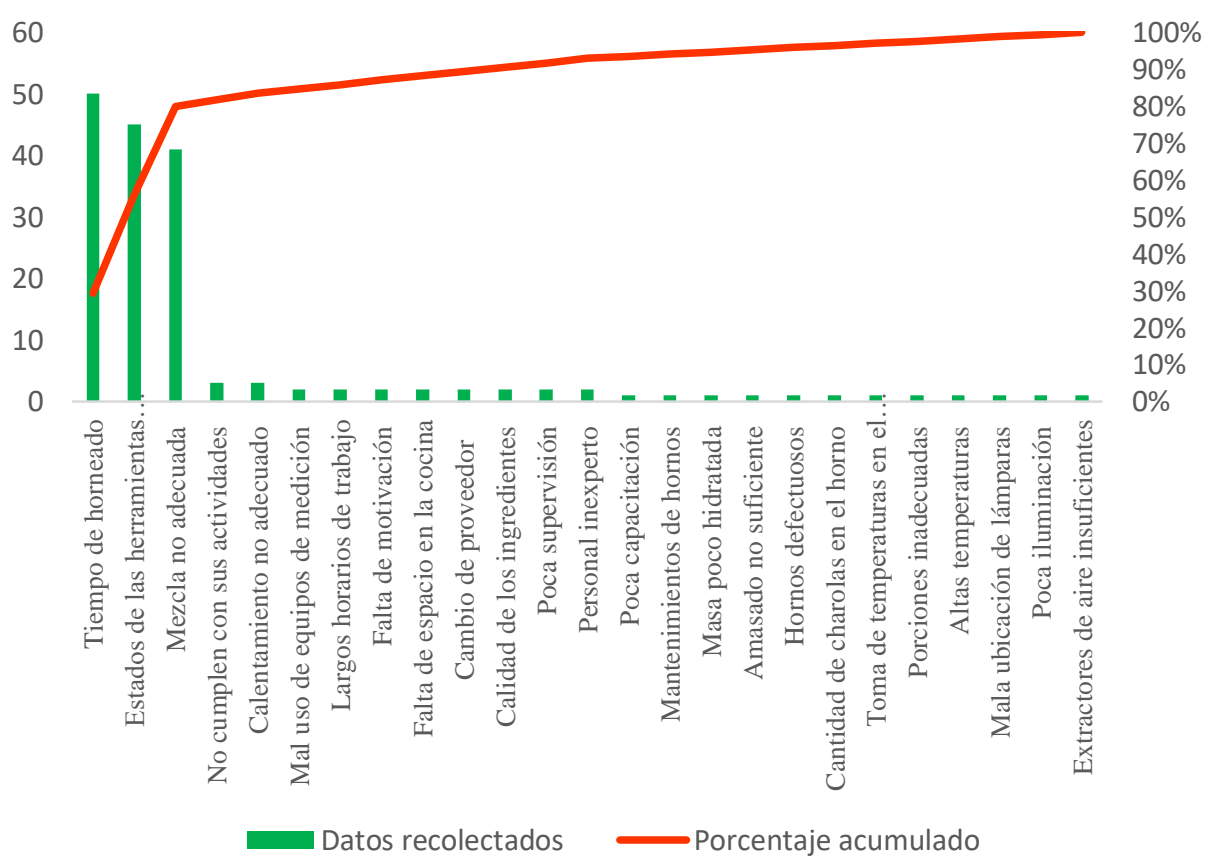


BILO Vol. 2 no. 1, Enero- Junio de 2020

Fig. 2 Causas del problema de la producción de panes. Fuente: Autores.

De acuerdo a la información obtenida por el diagrama de Pareto, se identificaron las causas de importante consideración para tener en cuenta en el proceso de la elaboración de panes, debido a que estos presentan defectos que se generan en el horneado. Inicialmente se conoce que el tiempo de horneado de los panes en esta panadería es de 50 minutos que es equivalente a 3000 segundos, que presenta una desviación de 120 segundos en la tabla 2, se presentan los datos utilizados para la realización de la carta X-R, tomando 10 muestras cada día por dos semanas.

\begin{tabular}{cccc}
\hline Formula & Dato & Formula & Dato \\
\hline $\mathrm{A} 2=$ & 0,308 & $\mathrm{LSE}=$ & 3,15 \\
$\mathrm{D} 3=$ & 0,223 & $\mathrm{LIE}=$ & 2,85 \\
$\mathrm{D} 4=$ & 1,777 & $\mu=$ & 3 \\
$\mathrm{LCS}=\mathrm{X}+(\mathrm{A} 2 * \mathrm{R})$ & 3104,855 & $\mathrm{LCS}=\mathrm{R} * \mathrm{D} 4$ & 669,847 \\
$=$ & & $\mathrm{LCI}=\mathrm{R} * \mathrm{D} 3$ & 84,061 \\
$\mathrm{LCI}=\mathrm{X}-(\mathrm{A} 2 * \mathrm{R})$ & 2872,651 & $\mathrm{R}=$ & 376,954 \\
$=$ & 2988,753 & $\mathrm{X}=$ &
\end{tabular}

Tabla 1. Especificaciones y límites para el diseño de la carta de control

Luego de realizar la carta de control, se procedió a construir los gráficos con los datos obtenidos como se muestra a continuación, se evidencia que el proceso se encuentra dentro de los límites de especificaciones, es decir, que el proceso está bajo control. En este caso se está controlando el tiempo de horneado de los panes en el que se muestra la estabilidad del proceso en el tiempo.

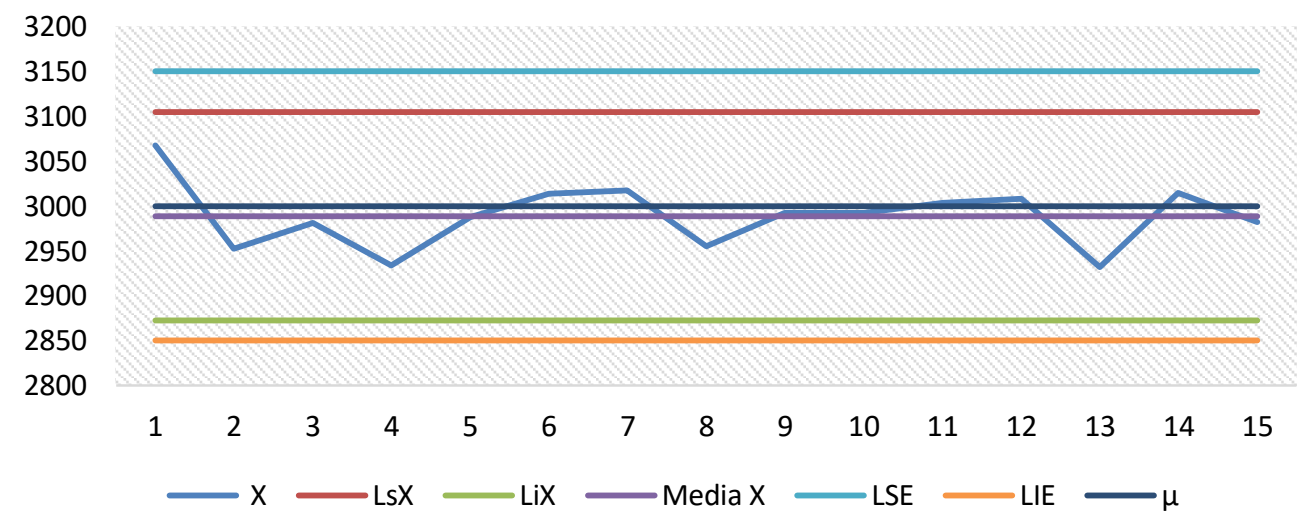

Fig. 3 Grafico de control X. Fuente: Autores.

En el grafico X, se muestran las especificaciones tanto de la carta como del cliente, las medias y los puntos se encuentran dentro de los límites y rondado las medias, lo cual es un aspecto positivo en cuanto a la estabilidad del proceso, ya que no presenta una variación significativa todo se encuentra dentro de las especificaciones.

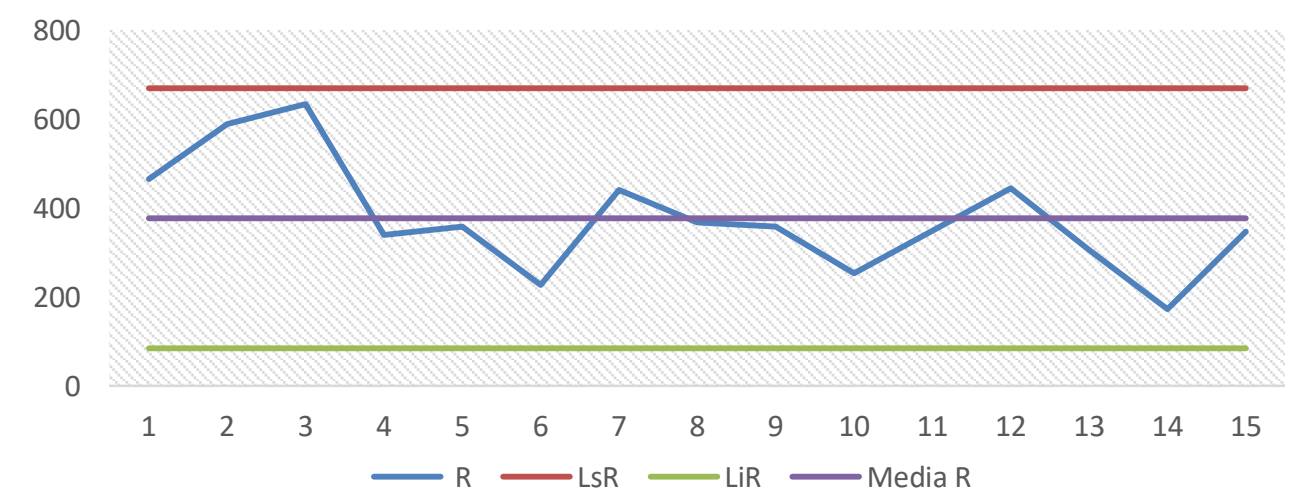

Fig. 4 Grafico de control R. Fuente: Autores. 
En el grafico R, se muestra una media de 376, límite superior de 669, límite inferior de 84 y sus puntos se encuentran alrededor de la media, sin embargo, existen un pico muy alto que casi toca el límite superior y un punto abajo que está muy cerca del límite inferior; sería pertinente tomar acciones para corregir esos puntos alejados de la media.

El factor más importante que impulsa al crecimiento de la economía de las microempresas son los clientes, es por esto que es valioso siempre revisar el estado de satisfacción de los mismos, para conocer sus preferencias de compra, lo que realmente desea en el producto que va a adquirir, el cliente requiere que le sea proporcionado un servicio y producto de calidad, con el fin de fidelizarlos y conseguir nuevos. Desde nuestro punto de vista, estas empresas deben incluir estrategias para mantener y aumentar la cantidad de clientes, estas deben ir guiadas por estudios de mercados. Es preciso decir que los mercados son muy cambiantes y se hace necesario estar muy pendientes de hacer estudios para conocer y movernos como se mueve el mercado para así avanzar a nivel económico y obtener posicionamiento por medio de la creación de valor.

Teniendo en cuenta la problemática que se venía presentando de los panes quemados, como se puede observar en los gráficos de control, existe un control. Sin embargo, se evidencian unos picos y es justo ahí donde se presenta el inconveniente. Corrigiendo este problema, se puede hacer cambio de la maquinaria (hornos) o realizarle mantenimientos más constantes y colocarle una configuración al tiempo que es verdaderamente necesario para que los panes se horneen completamente sin quemarse. Luego de hacer las respectivas correcciones en el proceso, para reactivar los clientes se deben toman acciones, pueden empezar por proporcionar nuevos productos, añadir valor a la producción, crear un ambiente atractivo, ofrecer líneas de producciones enfocadas a grupos especiales de clientes (línea de producción de panes sin gluten), gestionar en redes sociales, ser creativo en la generación de ofertas y el punto fundamental ser amables con los compradores y brindarles calidad.

\section{CONCLUSIONES}

Finalmente se concluyó que en todo negocio siempre se va a presentar una variabilidad en los procesos y en su paso a paso, es por ello que en los procesos productivos se puede encontrar algún producto defectuoso sin importar el método en él sea realizado. Ya haciendo referencia a el tema que se desarrolló en el artículo se evidencia cómo esta variación repercute en la pérdida de clientes, y como afecta esto a la empresa que es de servicio y dicho servicio es prestado a la comunidad; ahora si bien se observó que lo panes defectuosos son la razón perdida lo que se debe hacer es un reajuste al proceso y así obtener un buen control en el área de producción. Es importante resaltar que el uso de las herramientas de calidad es de gran importancia ya que ayuda a organizar las actividades por prioridades, dichas herramientas como las que se utilizaron en el desarrollo de este articulo como lo es el diagrama causa-efecto, el diagrama de Pareto, la carta de control X-R y los gráficos de control, ayudan a clarificar los problemas que se presentan en la empresa, en el momento en que se quiera obtener mejores resultados en un proceso.

Como sugerencia se le recomienda al negocio realiza una verificación con respeto a los tiempos de horneado del pan, ya que aquí es donde radica el mayor problema y por ello obtenemos un producto defectuoso el cual no es comprado por lo clientes ya que estos no se encuentran en buen estado, también sería bueno no solo revisar el proceso y sus tiempos, sino también la maquinaria para así estar seguros de que estas se encuentra en completamente adecuada para la cocción del pan. Ya que puede variar la estabilidad de horneado debido a que se encontró fugas tanto de gas como de aire caliente.

\section{Referencias}

[1] E.M. Saniga Technometrics, vol. 31, nº 2, pp. 313-320, 1989.

[2] R. d. 1. V. Humberto Gutierrez, «Herramientas basicas para seis sigma en control estadistico de calidad y seis sigma,» Mexico, 2004, p. 140.

[3] M. d. F. L. Maria Virginia Baptista, «Estrategias de lealtad de clientes en la banca universal,» Estudios gerenciales, vol. 29, $\mathrm{n}^{\circ} 127,2013$.

[4] A. K. a. C. S. McCracken, «Quality Technology and Quantitative Management,» vol. 10, nº 1, pp. 17-36, 2013.

[5] C. G. R. S. J. A. E. Pedro Ruiz Lopez, «Revista de calidad asistencial,» vol. 20, pp. 71 - 79. 
[6] F. C. L. H. R.C Quinino, «ScienceDirect,» enero 2020. [En línea]. Available: https://www.sciencedirect.com/science/article/pii/S036083521930600X. [Último acceso: 30 abril 2020].

[7] A. R. Saldaña, «SlideShare,» 16 mayo 2017. [En línea]. Available: https://www.slideshare.net/armand_29/aplicacin-de-las-7-herramientas-para-el-mejoramiento-de-la-calidad-delpan-hamburguesa-en-panaderia-america-s. [Último acceso: 23 abril 2020].

[8] L. L. J. a. K. C. I. Transactions, vol. 12, nº 2, pp. 182 - 195, 1981.

[9] M. d. F. L. Maria Virginia baptista, Abril - Junio 2013. [En línea]. Available: http://www.sciencedirect.com/science/article/pii/S0123592313000089. [Último acceso: 27 abril 2020].

Rodríguez, L., Castellano, M., \& Caridad, M. (2017). Planificación estratégica de recursos humanos en empresas [10] de consumo masivo. IJMSOR: International Journal of Management Science \& Operation Research, 2(1), 38-43. Recuperado a partir de http://ijmsoridi.com/index.php/ijmsor/article/view/84

[11] Bertolli, M., Roark, G., Urrutia, S., \& Chiodi, F. (2017). Revisión de modelos de madurez en la medición del desempeño. INGE CUC, 13(1), 70-83. https://doi.org/10.17981/ingecuc.13.1.2017.07 